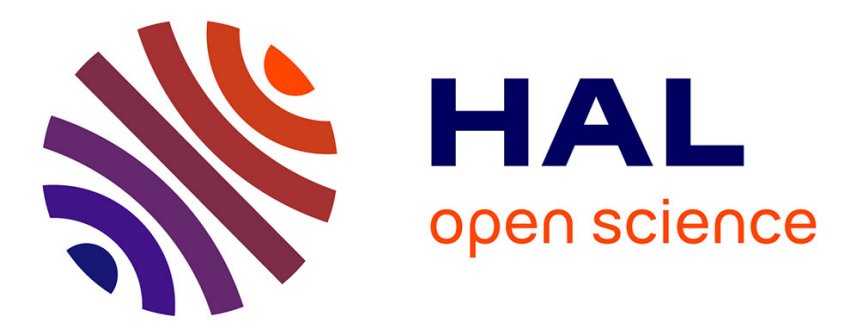

\title{
Spatio-temporal modelling of degradation processes through stochastic Gamma and Gaussian processes
}

Mestapha Oumouni, Franck Schoefs, Bruno Castanier

\section{To cite this version:}

Mestapha Oumouni, Franck Schoefs, Bruno Castanier. Spatio-temporal modelling of degradation processes through stochastic Gamma and Gaussian processes. 27th European Safety and Reliability Conference (Esrel 2017), 2017, Portoroz, Slovenia. pp.789-796, 10.1201/9781315210469 . hal-02525524

\section{HAL Id: hal-02525524 \\ https://univ-angers.hal.science/hal-02525524}

Submitted on 14 Aug 2020

HAL is a multi-disciplinary open access archive for the deposit and dissemination of scientific research documents, whether they are published or not. The documents may come from teaching and research institutions in France or abroad, or from public or private research centers.
L'archive ouverte pluridisciplinaire HAL, est destinée au dépôt et à la diffusion de documents scientifiques de niveau recherche, publiés ou non, émanant des établissements d'enseignement et de recherche français ou étrangers, des laboratoires publics ou privés. 


\title{
Spatio-temporal modelling of degradation processes through stochastic Gamma and Gaussian processes
}

\author{
M. Oumouni \& F. Schoefs \\ University of Bretagne Loire, Université de Nantes, Research Institute in Civil and Mechanical Engineering, \\ (GeM), UMR CNRS 6183, Nantes, France
}

B. Castanier

University of Bretagne Loire, Research Institute in Systems Engineering of Angers (LARIS), Université d'Angers, Angers, France

\begin{abstract}
In this work, we propose a new meta-model to describe the evolution of the degradation of infrastructures for reliability purposes. The model takes into account both hazards, temporal and spatial. It is based on the classical homogenous or non-homogenous Gamma process, the scale parameter is modeled with log-normal random field in order to assess and incorporate the effect of the spatial variability and heterogeneity on the degradation process.

Simulation results are performed using synthetic data based on Monte-Carlo simulations to fit the model, the inference of their parameters is performed using the method of moments combined with curve fitting method. Computation and estimation of quantities of interests for reliability or maintenance studies, namely the failure time and remaining life-time are developed and illustrated by numerical examples.
\end{abstract}

\section{INTRODUCTION}

Mathematical models based on partial differential equations with stochastic parameters and data are extensively studied in Mechanical and Civil Engineering $[1,8,2]$ to compute physical quantities varying in space and in time under uncertainty and spatial variability. However, their use in a reliability estimation context is faced with two major drawbacks.

Firstly, the approximation of such models to compute some physical quantities of interest can suffer from the curse of dimensionality, where it requires to solve a large number of deterministic problems. In particular when the variability and uncertainties are important $[1,6]$. Secondly, healthmonitoring data which are usually given by Non Destructive Techniques are not obviously linked to these models to update them with their associated parameters.

The Gamma process $[12,7,5]$ is widely used model for modeling degradation process encountered in civil engineering. However, it model only temporal variability and assume a uniform degradation on the whole structure and does not incorporate heterogeneity and spatial variability through component. On the other hand, to construct a complete degradation model with accurate predictions, a large amount of data using destruc- tive or nondestructive testing is required from a large amount of structures $[12,10,9,5]$ for predicting levels of the degradation with accuracy.

The major contribution detailed in this work is a new spatio-temporal random model based on Gamma process for predicting the degradation mechanism which takes into account both hazards, temporal and spatial. The temporal variability is modeled by the classical Gamma process and the spatial variability is modeled by a positive random field. This spatial field follows a log-normal distribution to describe the scaling parameter of the Gamma process. Under the assumption of stationarity satisfied by the random field, the spatial monitoring data of the component contributes in the parameters estimation to reduce uncertainty and increase the accuracy of the meta-model approach. Therefore, the method of moments based on the variogram curve fitting is used in the first stage to estimate the spatial parameters of the Gaussian field, in the second stage the method is reused to estimate temporal parameters.

The article is organized as follows, the spatialtemporal random field degradation model is presented in Section 2. The method of moments combined with curve fitting method are presented in Section 3 for identifying properties of the model in terms of statistical inference. Once the model is adjusted, Section 4 develops quantities of interests 
which are useful in the reliability and maintenance analysis, Namely, the failure time defined in terms of degradation level passages and remaining useful life time defined as the time of inspection of the unit to failure. Finally, Section 5 presents a numerical example of one dimensional variability illustrating the proposed methodology for model validation. Once the model is adjusted, Quantities of interest are developed and illustrated by an analytic and sampling approach.

\section{SPATIO-TEMPORAL DEGRADATION MODEL}

We look for modeling the degradation process by a convenient spatio-temporal random field to take into account its aleatory evolution with time and space. A separable model is one simple spatio-temporal model obtained through the tensorial product between a merely stochastic process $\left(X_{t}\right)_{t \geq 0}$ and a spatial random field $Z(z)$, where $z$ is the spatial variable. This class of separable random field is extensively used even in situations in which they are not always physically justifiable, since separability gives important computational and mathematic benefits.

The evolution in time models the intrinsic aleatory and the Gamma process is a natural candidate to catch this monotonous degradation. The spatial random field models the variability and uncertainty through the structure, and in many applications it is classically modeled by a second order stationary random field and given by a transformation of a Gaussian random field. Therefore, for simplicity and in order to construct a separable model, we assume that those source of randomness (time and space) are independent. Therefore, we consider a separable spatio-temporal random field to model the spatial variability in the degradation process.

Consider $\alpha(\cdot)$ to be a non-decreasing, rightcontinuous, real-valued function for $t \geq 0$ and vanishing at $t=0$ and $\beta>0$ a positive constant. A stochastic process $\left(X_{t}\right)_{t \geq 0}$ is said to be a Standard Gamma process with function shape $\alpha(\cdot)$ and identical scale parameter $\beta$ if it satisfies the following properties

- $X_{0}=0$ with probability one,

- $X_{t+s}-X_{t} \sim G a(\alpha(t+s)-\alpha(t), \beta)$,

- $X_{t}$ has independent positive increments,

Where $G a(\alpha(\cdot), \beta)$ is the Gamma distribution defined by the density function $f_{G a}(x)$ :

$$
f_{G a}(x)=\frac{\beta^{\alpha(\cdot)}}{\Gamma(\alpha(\cdot))} x^{\alpha(\cdot)-1} e^{-\beta x}, \text { for each } x>0,
$$

and $\Gamma$ is the classical Gamma function. The process $X_{t}$ satisfies the following scaling property,
$X_{t}=G a(\alpha(\cdot), 1) \beta^{-1}$, for each $\beta>0$.

The scaling property of the gamma process in (1) motivates us to consider the scale parameter to be a spatial random field to obtain a separable spatio-random filed model,

$G_{t}(z):=X_{t} \beta(z)^{-1}$,

where $\beta(\cdot)$ is spatial and positive random field which is assumed to be independent of $X_{t}$, and (without loss of generality we consider) $X_{t} \sim G a(\alpha(\cdot), 1)$. The scaling property satisfied by the process $X_{t}$ in (1) suggests to see (formally) the spatio-random field $G_{t}(z)$ as Gamma process with spatial random scale $\beta(\cdot)$.

In practice, it is difficult to verify and to find a positive distribution for a spatial or spatio-temporal random field. However, log-normal distribution occurs naturally as a limit distribution of physical processes, because the Central Limit Theorem applied to the product of positive independent random variables (number of measures $>30$ ) ensures that the log normal distribution can occur. Therefore, we choose the Log-normal distribution for the random scale coefficient,

$\beta(z)=e^{Y(z)}$,

where $Y$ is the spatial Gaussian random field $Y(z)$ which defined in $D$ a set in $R^{d}$ with $d=1,2,3$. The field $Y$ is assumed to be homogenous (stationary field) and then completely defined [14] by its constant mean $\mu:=\mathbb{E}[Y(\cdot)]$ and its stationary covariance function $\operatorname{cov}(r):=\mathbb{E}[Y(z+r) Y(z)]-\mu^{2}$.

The Matérn model of the covariance functions are the commonly used covariance in the engineering applications for the Gaussian random field. There are defined by the following function:

$\operatorname{cov}(r)=\sigma^{2} \frac{2^{1-\nu}}{\Gamma(\nu)}\left(\frac{\sqrt{2 v} r}{l_{c}}\right)^{v} K_{\nu}\left(\frac{\sqrt{2 v} r}{l_{c}}\right)$

where the parameters $\sigma^{2}, v, \alpha$ and $l_{c}$ are nonnegative real numbers, $\sigma^{2}$ is the variance of $Y, l_{c}$ is the correlation length, $r$ is the Euclidean distance between two points, $K_{v}$ denotes the modified Bessel function of the second kind. The parameter $v>0$ is a non-negative number that characterizes the degree of smoothness of cov which is related to the smoothness of the field $Y$. when $v=\frac{1}{2}$, cov coincides with the exponential covariance,

$c(r):=e^{-r / l} c$

this covariance is only Hölder continuous and so for the samples paths of $Y$. When $\nu \longrightarrow \infty$, it approaches the gaussian covariance, 
$c(r):=e^{-r^{2} /\left(2 l_{c}^{2}\right)}$,

which is an analytic function and so the samples paths of $Y$ are also analytic.

\section{METHOD OF MOMENTS FOR PARAMETERS INFERENCE}

In order to obtain a complete and accurate degradation model for practical examples, statistical methods for parameters estimation of gamma processes and gaussian random field are required. A typical data set of $G_{t}(z)$ consists of inspection points in different increasing time $t_{j}$ for $j=0, \ldots, N_{t}$ , with the same period $\tau$, where for each time $t_{\text {t }}$ the inspection positions are given in uniform positions $z_{l}$ for $l=1, \ldots, N_{z}$ with step size $h$. Here, we assume for simplicity that $\alpha(\cdot)$ follows a power law:

$$
\alpha(t)=a t^{b},
$$

for some unknown $a>0$ and a known power $b=1$.

\subsection{Step 1: Spatial parameters}

The Method of Moments (MOM) uses the benefit of the separability of the model to estimate the parameters of $G_{t}(z)$ in two steps. The first step of (MOM) consists in estimating the spatial parameters of the model. They are defined as the parameters of the second order stationary random field $Y$, i.e, the variance $\sigma^{2}$, the correlation length $l_{c}$ and the regularity parameter $v$ of the correlation function given in (3).

In order to obtain an estimate of these parameters, we use nonparametric estimate of the semivariogram which is typically obtained through (MOM) of one or more realizations of $Y$ and defined by [3]:

$$
\hat{\Upsilon}_{Y}\left(h_{l}\right)=\frac{1}{2 N_{h_{l}}} \sum_{z_{i}, z_{j} \in S_{h_{l}}}\left(Y\left(z_{i}\right)-Y\left(z_{j}\right)\right)^{2},
$$

where $S_{h}$ is the set of the points separated with distance $h_{l}$ and $N_{h_{l}}$ its cardinal.

For a fixed time instant $t$, the variogram of the field $\log \left(G_{t}(\cdot)\right)$ equals the variogram of $Y(\cdot)$. Therefore, the empirical variogram $\hat{\Upsilon}_{Y}$ is obtained by computing the empirical variogram of the random field $\log \left(G_{t}\right)$, the logarithm of $G_{t}$. Thus, in any fixed time $t$ we get:

$$
\hat{\Upsilon}_{Y}\left(h_{l}\right)=\frac{1}{2 N_{h_{l}}} \sum_{z_{i}, z_{j} \in S_{h_{l}}}\left(\log \left(G_{t}\left(z_{i}\right)\right)-\log \left(G_{t}\left(z_{j}\right)\right)\right)^{2}
$$

When $M$ realizations of the degradation $G_{t}(z)$ are available, they can be used to improve the estimation of $\hat{\Upsilon}_{Y}\left(h_{l}\right)$ by empirical average.

Since the random field $Y$ is stationary, the exact variogram is given by:

$$
\Upsilon_{Y}\left(h_{l}\right)=\sigma^{2}-\sigma^{2} \operatorname{cov}\left(h_{l}\right),
$$

where the correlation function $\operatorname{cov}(h)$ is given in (3). Therefore, we estimate the spatial parameters by minimizing the quadratic error (Least square method) between exact variogram and experimental one, thus $\sigma^{2}, l c$ and $v$ are deduced by the following minimization problem.

$$
\min _{\sigma, l, v>0} \sum_{l=1}^{N_{z}}\left(\hat{\Upsilon}_{Y}\left(h_{l}\right)+\sigma^{2} \operatorname{cov}\left(h_{l}\right)-\sigma^{2}\right)^{2}
$$

Remark 3.1. The classical least square method can be extended to a generalized least squares (GLS) method, where we minimize a weighted error, given by the correlation matrix $R$ of the set $\left\{\hat{\Upsilon}_{Y}\left(h_{l}\right)\right\}_{l=1}^{N_{z}}$. A simplified approach of this generalization is the weighted method where the matrix $R$ is diagonal with entries $R_{l}=\frac{2 r_{Y}\left(h_{l}\right)}{N_{h_{l}}}$ as suggested in [3] assuming a Gaussian law and non-correlation among $\left\{\hat{\Upsilon}_{Y}\left(h_{l}\right)\right\}_{l=1}^{N_{z}}$.

We note when the space positions are not equidistant, estimation (5) is slightly modified for non uniform grid to compute the experimental variogram of $Y$ from the logarithm of the field $G_{t}(\cdot)$, spatial mean is given on all pairs of points whose distance are between $h$ and $h+\delta h$ for some threshold $\delta h>0$ [3].

\subsection{Step 2: Temporal parameters}

The temporal parameters are defined as the parameters of the process $X$, i.e, the shape function $\alpha(t)$ defined in (4) and the deterministic contribution $\eta=e^{\mu}$ of the scale field $\beta$. Since we assume that the power $b$ is known, we estimate then $a$ and $\eta$. Note that $\eta$ contains a contribution of the spatial mean of the random field $Y$. Let $\left\{G_{t, i}^{i}\left(z_{l}\right)\right\}$ be a sequence of independent and identically distributed (i.i.d.) simulations of the degradation model described in the previous section. Each $i$-th degradation process is observed at time $t_{j}$ among $N_{t}$ times and on location $x_{l}$ among $N_{z}$ locations.

Logarithm of increments of sample paths of $G_{t}(z)$ writes:

$\xi_{j, l}^{i}:=\log \left(\delta G_{t_{j}}^{i}\left(z_{l}\right)\right)=\log \left(X_{t_{j}}^{i}-X_{t_{j-1}}^{i}\right)+Y^{i}\left(z_{l}\right)$,

for $i \in[1, M], j \in\left[1, N_{t}\right], l \in\left[1, N_{z}\right]$.

Since the process $X_{t}$ and the field $Y$ are independent, the first two moments of $\xi_{j, l}^{i}$ are given by: 
$m_{1}:=\mathbb{E}\left[\xi_{j, l}\right]=\psi\left(a \tau_{j}\right)-\log (\eta)$

$\left.m_{2}:=\operatorname{var}\left[\xi_{j, l}^{i}\right]=\psi_{1}\left(a \tau_{j}\right)\right)+\sigma^{2}$,

where the function $\psi$ is digamma function which is defined as the logarithmic derivative of gamma function $\Gamma$, and $\psi_{1}$ is the trigamma function defined as the derivative of $\psi$. The temporal grid being uniform, thus increments $\left(X_{t_{j}}^{i}-X_{t_{j-1}}^{i}\right)_{j=1}^{N_{t}}$ are independent and identically distributed. From $M N_{t}$ realizations of those increments combined with spatial average we estimate $m_{1}$ and $m_{2}$ by the following sum:

$m_{1} \approx\left(M N_{z} N_{t}\right)^{-1} \sum_{i=1}^{M} \sum_{j=1}^{N_{t}} \sum_{l=1}^{N_{z}} \xi_{j, l}$

$m_{2} \approx\left(M N_{z} N_{t}\right)^{-1} \sum_{i=1}^{M} \sum_{j=1}^{N_{t}} \sum_{l=1}^{N_{z}}\left(\xi_{j, l}\right)^{2}-m_{1}^{2}$.

Therefore, an estimation of temporal parameters $a$ and $\eta$ is given by:

$$
\left(\begin{array}{l}
a \\
\eta
\end{array}\right)=f^{-1}\left(\begin{array}{l}
m_{1} \\
m_{2}
\end{array}\right)
$$

where we define the function $f$ by

$$
f\left(\begin{array}{l}
u \\
v
\end{array}\right)=\left(\begin{array}{c}
\psi(u \tau)-\log (v) \\
\psi_{1}(u \tau)+\hat{\sigma}^{2}
\end{array}\right)
$$

with $\tau=t_{j}-t_{j-1}$ and $m_{1}$ et $m_{2}$ are estimated in (11, 12) and $\hat{\sigma}^{2}$ is the estimate variance $\sigma^{2}$ obtained in equation (8).

Remark 3.2. When $b$ is unknown, we can use the approach of pseudo maximum-likelihood method (PML) to estimate temporal parameters. It consists of maximizing the likelihood of the sequence of the increment $\left(\delta_{1} G, \&, \delta_{t_{N}} G\right)$ on a given fixed spatialposition $z$. These increments are conditionally independent and their likelihood is given by the product of the marginal density of the field $G_{t}(z)$. This marginal density is approximated in Section 4 (equation (15) and (16)).

Method of moments for temporal parameters can be extended when $b \neq 1$, the non-stationary Gamma process can be easily transformed to a stationary one by performing a monotonic transformation $u(t)=t^{b}$ on the time increments ( see [12] for more details).

\section{QUANTITIES OF INTEREST}

\subsection{Marginal density approximation}

The marginal distribution noted by $f_{t}(\cdot)$ of the model $G_{t}(z)$ does not depend on the position $z$ but only on the time $t$, because the spatial random field $Y$ is homogenous. This distribution can be constructed numerically by simulating paths ofthe field $G_{t}(z)$. It is useful in computation of some quantities of interest which are used in structural reliability and maintenance. We can also use this distribution as a pseudo-likelihood of $G_{t}(z)$ to conclude an estimation of the temporal parameters of the model.

Let $\xi(y)$ be the density of the standard gaussian random variable $N(0,1)$. By using the fact that the temporal paths of increments of the model $G_{t}(\cdot)$ are conditionally independent, we compute the distribution $f_{t}$ for all $v>0$ by the following form:

$$
f_{t}(v)=\psi_{t}(v) \int_{\mathbb{R}} \exp (-v \eta \exp (\sigma y)+\alpha(t) \sigma y) \xi(y) d y,
$$

where we set $\psi_{t}(v):=\frac{\eta^{\alpha(t)} v_{v}(\alpha(t)-1)}{\Gamma(\alpha(t))}$.

The integral in (15) has a transcendental form, thus we use Gauss-Hermite quadrature formula to approximate this marginal density $f_{t}$.

We consider $m$ roots $\left\{y_{j}\right\}_{j=1}^{m}$ of the Hermite polynomial and their associated weights $\left\{w_{j}\right\}_{j=1}^{m}$. Thus, an approximation of $f_{t}$ writes:

$$
f_{t}^{m}(v):=\psi_{t}(v) \sum_{j=1}^{m} \exp \left(-v \eta \exp \left(\sigma y_{j}\right)+\alpha(t) \sigma y_{j}\right) w_{j}
$$

The convergence of the sequence $f_{t}^{m}(v)$ for any fixed positive reel $v>0$ is relatively fast, since the integrand is infinitely differentiable. However the norm of any $m$-derivative of this integrand depends on the value of the parameters $\alpha(t), \sigma$ and $\eta$. A large value of these parameters requires a large order $m$ of the approximation in (16), in particular for large time $t$. Therefore, the order of the approximation $m$ is established by the following stop criterion,

$$
\left|f_{t}^{m}-f_{t}^{m-1}\right| \leq \mathcal{E}
$$

where $\varepsilon>0$ is a convenient threshold value. Note that when $v \rightarrow \infty$ both $f_{t}(v)$ and $f_{t}^{m}(v)$ decreases to zero.

\subsection{Distribution of failure time}

The failure time $T_{F}$ for a structural component is defined as the time at which the degradation path $G_{t}$ first crosses a critical level $g_{F}$ for any spatial location,

$T_{F}=\inf \left\{t>0 ; G_{t}(\cdot) \geq g_{F}\right\}$,

In what follows, the critical level $g_{F}$ is assumed deterministic. For some simple path models, the distribution of $T_{F}$ defined by $F_{T}(t):=P\left(T_{F}<t\right)$ can 
be expressed in a closed form. However, this is not always possible and it can be numerically computed using Monte Carlo simulations by simulating paths of $G_{t}(z)$. The sample paths of $G$ are monotonic since the spatial variability $e^{Y}$ is positive, thus the failure time cumulative distribution function $F_{T}(T)$ satisfies:

$$
F_{T}(t)=1-P\left(T_{F}>t\right)=1-\int_{0}^{g_{F}} f_{t}(z) d z,
$$

where $f_{t}$ is the marginal pdf of $G_{t}$ given in (15). Therefore, using the approximation $f_{t}^{m}$ with a convenient order $m$ we approximate the distribution $F_{T}$,

$$
F_{T}^{m}(t)=1-\int_{0}^{g_{F}} f_{t}^{m}(v) d v,
$$

The integral (18) can be computed accurately by any quadrature formula, for example the LegendeGauss quadrature.

The derivative of (17) and (18) with respect to the time $t$ provides the probability density function of $T_{F}$ and its approximation respectively.

The approximation of the cumulative density (18) requires a quite huge cost when $t$ or $\sigma^{2}$ is large. In this case one can construct an estimation of $F_{T}$ by generating a sufficiently large number of random sample paths of $G_{t}(z)$ with estimated parameters using Monte-Carlo (MC) simulations.

By fixing $N_{t}$ desired times, $N_{z}$ desired locations and $M$ realisations of spatio-temporal paths $\left\{G_{t}^{m}\left(z_{j}\right)\right\}$ for $i=1 \&, N_{t}, j=1 \&, N_{z}$ and $m=1, \&, M$. The estimate $\tilde{F}_{T}$ of $F_{T}$ is given by,

$$
\tilde{F}_{T}\left(t_{i}\right):=\frac{\sum_{j, m}^{N_{z}, M} I_{\left\{G_{i}^{m}\left(x_{j}\right) \geq g_{F}\right\}}}{M N_{z}},
$$

where $\mathbb{I}_{A}$ represents the characteristic function of the set $A$, i.e $\mathbb{I}_{A}(z)=1$ if $z \in A$ and zero otherwise.

Note that since the random field $G_{t}$ is homogeneous with respect to the spatial variables, the estimate $\tilde{F}_{T}$ can be computed also using realizations of $G_{t}\left(z_{p}\right)$ fixed at any position $z_{p}$,

$$
\tilde{F}_{T}\left(t_{i}\right) \approx \frac{\sum_{m}^{M} \mathbb{I}_{\left\{G_{i}^{m}\left(z_{p}\right) \geq g_{F}\right\}}}{M} .
$$

However the estimation (20) needs to use more MC simulations of $G_{t}(z)$ than (19) since by the ergodic property, the spatial average contributes to the convergence of $\tilde{F}_{T}$ to $F_{T}$.

\subsection{Remaining useful lifetime after inspection}

In reliability analysis and survival studies, residual lifetime after inspection is a key indicator. In the maintenance decision analysis, the current measured degradation is used to predict the remaining useful lifetime (RL) of the structure [11]. If $t$ is the current time of inspection, the residual lifetime is defined by the random variable:

$$
R L_{t}:=\inf \left\{\tau>0, G_{t+\tau} \geq g_{F} \mid G_{t}=g_{t}\right\},
$$

where $g_{F}$ is the critical level and $g_{t}$ is the measured degradation at given time $t$, implicitly $g_{t}<g_{F}$. When we suppose that a component has survived to a given time $t$ and we have not any information or measure about the current degradation path $G_{t}$, then a conditional reliability function gives an evaluation of the remaining lifetime:

$$
\begin{aligned}
R\left(\tau \mid T_{F}>t\right) & :=P\left(T_{F} \geq \tau+t \mid T_{F}>t\right) \\
& =\frac{\int_{0}^{g_{F}} f_{\tau+t}(y) d y}{\int_{0}^{g_{F}} f_{t}(y) d y}
\end{aligned}
$$

When the current degradation measure path of $G_{t}$ is available, then by conditioning of the failure time would give a more accurate prediction than (21), i.e the probability that the unit survival after the time $t+\tau$ given its actual state $G_{t}=g_{t}$ at time $t$ is:

$$
\begin{aligned}
P\left(R L_{t}>\tau\right) & =P\left(G_{t+\tau}<g_{F} \mid G_{t}=g_{t}\right) \\
& =P\left(\delta_{\tau} G_{t}<g_{F}-g_{t} \mid G_{t}=g_{t}\right),
\end{aligned}
$$

where we define the increment $\delta_{\tau} G_{t}:=G_{t+\tau}-G_{t}$. If we note by $f_{\delta_{\tau} G_{t} \mid G_{t}}$ the conditional marginal density of the process $\delta_{\tau} G_{t}$ given the event $\left\{G_{t}=g_{t}\right\}$, then we compute the probability cumulative distribution $F_{R L}:=P\left(R L_{t} \leq \tau\right)$ of the residual lifetime by:

$$
\begin{aligned}
P\left(R L_{t} \leq \tau\right) & =1-P\left(\delta G_{t}<g_{F}-g_{t} \mid G_{t}=g_{t}\right) \\
& =1-\int_{0}^{g_{F}-g_{t}} f_{\delta_{\tau} G_{t} \mid G_{t}}(y) d y .
\end{aligned}
$$

Using the independence of the increments of Gamma process, we compute the conditional density of $\delta_{\tau} G_{t}$ given $G_{t}=g_{t}$ by,

$$
f_{\delta_{\tau} G_{t} \mid G_{t}}(u)=\frac{u^{\delta_{\tau} \alpha-1} g_{t}^{\alpha(t)-1} f_{\tau+t}\left(u+g_{t}\right)}{B\left(\delta_{\tau} \alpha, \alpha(t)\right)\left(u+g_{t}\right)^{(\alpha(\tau+t)-1)} f_{t}\left(g_{t}\right)}
$$

where we set $\delta_{\tau} \alpha:=\alpha(t+\tau)-\alpha(t), f_{t}$ is the marginal density of $G_{t}$ given in $(15)$ and $B(x, y)=\frac{\Gamma(x) \Gamma(y)}{\Gamma(x+y)}$ is the beta function. It follows that $F_{R L}(\tau)$ is given by,

$F_{R L}(\tau)=1-\int_{0}^{g_{F}-g_{t}} f_{\delta_{\tau} G_{t} \mid G_{t}=g_{t}}(u) d u$.

By using an appropriate approximations of densities $f_{t+\tau}^{m}\left(u+g_{t}\right)$ and $f_{t}^{m}\left(g_{t}\right)$ we get $f_{\delta_{-} G_{t} \mid G_{t}=g_{t}}^{m}(u)$ an approximate of the conditional probability density in (24) and then an approximation of $F_{R L}(\tau)$. 
Note that when $t=0$ and $g_{t}=0$, equation (24) is not definite and the cumulative probability function $F_{R L}$ is the function $F_{T}$ given in (17).

The derivative of (23) with respect to the variable $\tau$ provides the probability density function of the Residual lifetime $R L$.

\section{NUMERICAL ILLUSTRATION}

In this section we simulate the degradation model through Monte Carlo simulations to validate the inference process and the approximation of the quantities of interest.

We consider $M=1,10,100$ sample paths of the field $\left(G^{j}\right)_{j=1}^{M}$, each trajectory $G^{j}$ being simulated at $N_{t}$ equidistant periods on the interval time [0, 30] (in years) and $N_{z}$ equidistant locations in one dimensional and two dimensions space.

The model is stationary with respect to time, i.e the shape parameter is linear $\alpha(t)=a t$, the deterministic contribution of the scale random field is given by $\eta=e^{\mu}$ where $\mu=2 / 3$ (mean of $Y$ ), $\sigma^{2}=0.6$ (variance of $Y$ ), $l_{c}=1$ (correlation length) and $\nu=2$ (smoothness parameter of $Y$ ).

The Gaussian random field is defined on the interval $[0, L]$ where $L=100$. We use the method of circulant matrix [4] to simulate $Y$ with exact discretization in $N_{z}$ equidistant spatial positions.

In what follows, for simplicity, a fixed value of the smoothness parameter $\nu=2$ is selected (i.e the paths of $G$ are one time derivative in quadratic norm).

Figure 1 (left) plots one realization of the model $G_{t}(z)$ and compares (right) its experimental variogram with exact one of the Gaussian field $Y$. The experimental variogram is computed using the spatial trajectory of the logarithm $\log \left(G_{t}(z)\right)$ at time $\mathrm{t}=30$.

\section{Method of moments (MOM) Step 1}

The first step of MOM consists in estimating spatial parameters, variance $\sigma^{2}$ and correlation length
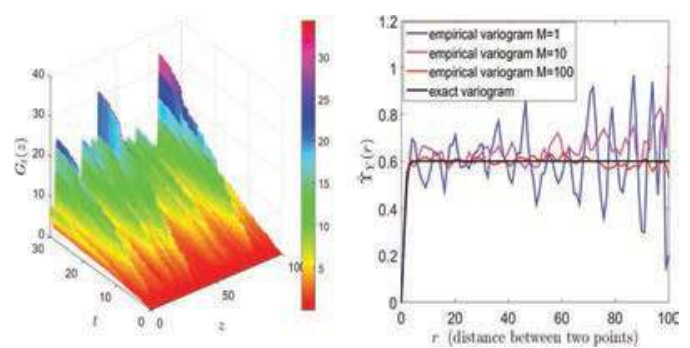

Figure 1. Left: example of one realization of $G_{t}(\cdot)$, right: variogram $\left(l_{c}=1, \nu=2, \sigma^{2}=0.6\right)$.
Table 1. Parameters estimation of one dimensional spatial variability by MOM.

\begin{tabular}{lll}
\hline$N_{z}=40$ & & \\
\hline$M$ & $\sigma^{2}$ & $l_{c}$ \\
\hline 1 & $0.714(0.114)$ & $0.21(0.791)$ \\
10 & $0.5720(0.028)$ & $0.81(0.19)$ \\
100 & $0.6048(0.0048)$ & $0.890(0.1024)$ \\
\hline$N_{z}=100$ & & \\
\hline$M$ & & $l_{c}$ \\
\hline 1 & $\sigma^{2}$ & $1.186(0.186)$ \\
10 & $0.546(0.053)$ & $0.934(0.065)$ \\
100 & $0.584(0.015)$ & $1.024(0.024)$ \\
\hline
\end{tabular}

$l_{c}$. The quality of the estimation is measured by the mean absolute errors which is given by the average of absolute difference between the exact parameter and 10 estimated values calculated across MOM.

Table 1 gives estimates of those parameters obtained by MOM where we minimize equation (8) with $\nu=2$ and three values of $M$. (Mean absolute errors in brackets).

From Table 1, the estimation of the correlation length $l_{c}$ depends strongly on the number of spatial positions $N_{z}$, obviously because $l_{c}$ is very small with respect to the length $L=100$. In contrast of $l_{c}$, the estimate of the variance $\sigma^{2}$ is largely acceptable even with small number of positions and with only one realization of $G_{t}(\cdot) \quad\left(M=1, N_{z}=40\right)$. However, the estimation of quantities of interest depends strongly on the variance $\sigma^{2}$, so an accurate estimate of $\sigma^{2}$ is needed to forecast reliable predictions.

\section{Method of moments Step 2}

Once spatial parameters are estimated, the second step of the MOM consists in estimating the temporal parameters, which are given by (13). An estimation of the variance $\sigma^{2}$ is inserted in (13) for each case of $M$ and $N_{z}$. Table 2 summarizes estimations of temporal parameters $a$ and $\eta$. Results show that their accuracy depends strongly on the total inspection times $N_{t}$ and on the total number of positions $N_{z}$. An acceptable accuracy is reached for small number of realizations $M(M=10)$ when $N_{t}$ and $N_{z}$ are significatively large $\left(N_{t}=60, N_{z}=40\right)$. In particular, estimate of $\eta$ depends significatively on the number of locations $N_{z}$ since it contains the stochastic contribution of the random field $Y$.

Figure 2 illustrate the convergence of the MOM for temporal parameters $\alpha$ and $\eta$, where we consider a database with size $M \times N_{z} \times N_{t}$ of the 
Table 2. Parameters estimation of temporal variability using MOM.

\begin{tabular}{lll}
\hline$N_{z}=40, N_{t}=30$ & \\
\hline$M$ & $a$ & $\eta$ \\
\hline 1 & $0.79(0.21)$ & $1.487(0.46)$ \\
10 & $0.947(0.053)$ & $2.157(0.21)$ \\
100 & $1.012(0.012)$ & $1.986(0.038)$
\end{tabular}

\begin{tabular}{lll}
\hline$N_{z}=40, N_{t}=60$ & \\
\hline$M$ & $A$ & $\eta$ \\
\hline 1 & $0.902(0.098)$ & $1.617(0.33)$ \\
10 & $1.027(0.027)$ & $2.087(0.14)$ \\
100 & $1.01(0.009)$ & $1.97(0.022)$ \\
\hline
\end{tabular}

\begin{tabular}{lll}
\hline$N_{z}=100, N_{t}=30$ & \\
\hline$M$ & $a$ & $\eta$ \\
\hline 1 & $0.817(0.18)$ & $2.377(0.43)$ \\
10 & $1.057(0.057)$ & $1.837(0.11)$ \\
100 & $1.012(0.012)$ & $2.015(0.068)$ \\
\hline
\end{tabular}

\begin{tabular}{lll}
\hline$N_{z}=100, N_{t}=60$ & \\
\hline$M$ & $a$ & $\eta$ \\
\hline 1 & $1.01(0.1)$ & $1.567(0.38)$ \\
10 & $1.029(0.029)$ & $1.817(0.13)$ \\
100 & $1.01(0.009)$ & $2.973(0.026)$ \\
\hline
\end{tabular}
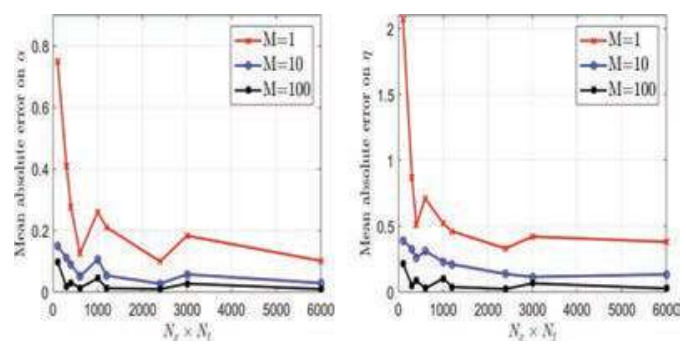

Figure 2. Mean absolute error on $\alpha$ (left) and $\eta$ (right).

simulated model $G$. The mean absolute error on $\alpha$ and $\eta$ is given as function of the product $N_{z} \times N_{t}$ where $N_{z}=10,40,100$ and $N_{t}=10,30,60$.

We remark a strong improvement for the estimation for $M>1, N_{z}>10$ and $N_{t}>10$. Estimate accuracy of $\alpha$ is nearly independent of the spatial positions unlike $\eta$ where accuracy depends on $N_{z}$ and strongly on $N_{t}$. An acceptable accuracy is reached in the case of one component $M=1$ when $N_{t}$ and $N_{z}$ are quite large $\left(N_{t}=60, N_{z}=40\right)$. A large number of positions $N_{z}$ does not improve the convergence of these temporal parameters unlike spatial parameters.

Once parameters estimation of the model is performed, we accurately simulate $G$ at any position
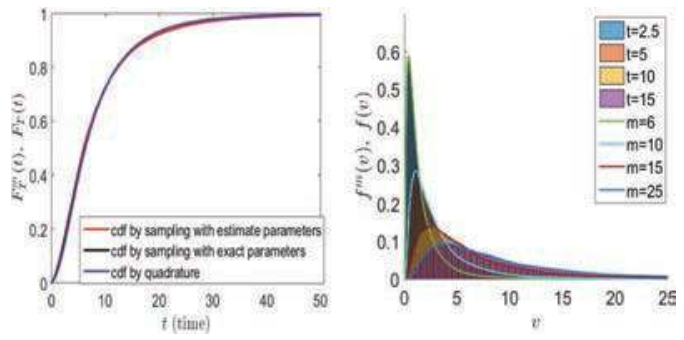

Figure 3. Left: estimate of the failure time distribution $F_{T}$, right: marginal density by sampling and quadrature approach.
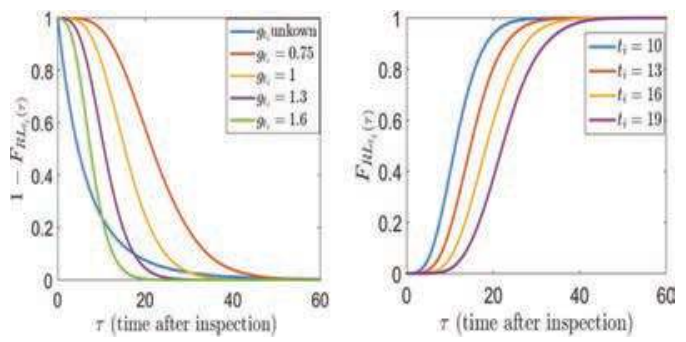

Figure 4. Left: Distribution of predictive time of inspection to failure, right: reliability and remaining lifetime function.

and time. Therefore, in order to estimate the marginal cumulative distribution $F_{T}$ of failure time $T_{F}$ by sampling method (19), we perform $10^{3} \mathrm{MC}$ simulations of $G_{t}(\cdot)$ discretized on $N_{t}=100$ times and $N_{z}=100$ positions. We plot in Figure 3 (left) the cdf $F^{T}$ computed by sampling approach with exact and estimated parameters and by quadrature rule (18) with $m=30$ Gaussian knots.

Figure 3 (right) compares marginal density of $G_{t}$ by sampling and by quadrature given in (16) at several times $t$ with a convergence criterion $\mathcal{E} \approx 10^{-3}$. We note that the required order of the quadrature rule increases with time $t$.

Figure 4 (left) illustrates the effect of conditioning the current state on the failure prediction at the current time of inspection $t_{i}=10$ for several measured degradations levels from 0.75 to 1.6 .

The survival function depends on the current state and gives more prediction than the reliability function ( $g_{t_{i}}$ is unknown, blue curve). The curves highlight how the reliability function underestimates or overestimates the time to failure under thevalue of $g_{t_{i}}$. In Figure 4 (right), we draw several failure curves of the remaining lifetime $F_{R L_{t}}(\tau)$ with several times of inspection $t_{i}$ with the same condition state $g_{t_{i}}=1.25$. This figures highlight an obvious result, the more the time for observing given condition state, the more the residual lifetime. 


\section{CONCLUSION}

In this paper, we have developed a spatio-temporal degradation model that incorporates the spatial variability and heterogeneity across structural component. It is based on the basic gamma process with a scale parameter modeled with nonnegative spatial random field. The temporal paths of the process are monotonic with conditionally independent increments, the positive random field scale follows a log-normal distribution as limit of independent positive variables.

The quantities of interest that are useful in reliability analysis and in maintenance, namely the distribution of failure time and the distribution of remaining useful lifetime are computed. A Method of moments is carried out to infer the spatial and temporal parameters of the model. Monte Carlo simulations illustrate the advantage of the proposed model. The advantages of the proposed model are that uncertainties are reduced and the accuracy of the inference is improved by exploiting with batter manner the spatial data.

An interesting extension of the current model can consider the bivariate modeling based on the state dependent Gamma process.

\section{ACKNOWLEDGEMENTS}

The work reported in this article was supported by the Region Pays de la Loire de France through the regional project SI3M (Strategies of Identification of Meta-models for Structural Maintenance). The authors would like to thank the region for the support.

\section{REFERENCES}

[1] I. BABuŠKA, R. Tempone, AND G. Zouraris. (2005) Solving elliptic boundary value problems with uncertain coefficients by the finite element method: the stochastic formulation. Comput. Methods Appl. Mech. Engrg., 194(12-16), 1251-1294.
[2] Behrouz Shafei, Alice Alipour. (2015). Application of large-scale non-Gaussian stochastic fields for the study of corrosion-induced structural deterioration, Engineering Structures, 88, 262-276.

[3] Cressie, N. (1993). Statistics for Spatial Data, Wiley, New York.

[4] Dietrich, C. et G. Newsam. (1997). Fast and exact simulation of stationary Gaussian processes throught circulant embedding of the covariance matrix. SIAM J. SCI. COMPUT. Vol. 18, No. 4, 1088-1107.

[5] El Hajj, Boutros, Bruno Castanier, Franck Schoefs, and Thomas Yeung. (2015). A Maintenance-Oriented Degradation Model for a Reinforced Concrete Structure Subject to Cracking. Journal of Risk and Reliability, Part O, 1-13.

[6] J. Erhel, Z. Mghazli, M. Oumouni. (2015). An adaptive sparse grid method for elliptic PDEs with stochastic coefficients Computer Methods in Applied Mechanics and Engineering, 297, 392-407.

[7] LAWless J, Crowder M. (2004). Covariates and random effects in a gamma process model with application to degradation and failure. Lifetime Data Anal; 10(3), 213-227.

[8] Papakonstantinou KG, Shinozuka M. (2013). Probabilistic model for steel corrosion in reinforced concrete structures of large dimensions considering crack effects. J Eng Struct, 57, 306-326.

[9] F. Schoefs, E. Bastidas-Arteaga, T.V. Tran, G. Villain B, X. Derobert. (2016). Characterization of random fields from NDT measurements: A two stages procedure. Engineering Structures, 111, 312-322.

[10] Schoefs F, Clement A, Nouy A. (2009). Assessment of spatially dependent ROC curves for inspection of random fields of defects. Struct Saf, 31, 409-419.

[11] Si, Xiao Sheng, Wenbin Wang, Chang Hua Hu, AND Dong Hua Zhou. (2011). Remaining Useful Life Estimation - A Review on the Statistical Data Driven Approaches. European Journal of Operational Research, 213, Issue 1, 1-14

[12] Van NoortwiJk, J.M. (2009). A Survey of the Application of Gamma Processes in Maintenance. Reliability Engineering \& System Safety, 94 2-21.

[13] Vu K, Stewart M. (2005) Predicting the likelihood and extent of reinforced concrete corrosion-induced cracking. J. Struct. Eng., Volume 131, Issue 11, 1681-1689.

[14] A.M. YAGLOM, (1987) Correlation Theory of Stationary and Related Random Functions, SpringerVerlag, New York. 